\title{
Grand Challenges in Physical Oceanography
}

\author{
Hans van Haren* \\ Royal Netherlands Institute for Sea Research and Utrecht University, Den Burg, Netherlands
}

Keywords: physical oceanographic processes, extreme events research, coupling between large and small scales, observation techniques development, modeling issues

\section{INTRODUCTION}

Physical oceanography is a relatively young scientific branch, in comparison with marine biology or other oceanographic disciplines, and with most physics disciplines. However, because of importance for fisheries and trading, surface ocean physics on currents and waves has been investigated for over centuries. It was largely applied science; a lot of fundamental science on the ocean interior is probably still ahead of us. This may have to do with the ocean's inaccessibility for mankind. Some challenges are given here, based on a biased diagnosis of present-day shortcomings in physical oceanography research.

\section{PRESENT-DAY PROBLEMS}

The physical environment and variations in its governing processes have direct impact on sea-life, economics, atmospheric, and climate science, fluid dynamics issues and all general oceanographic disciplines. This may not be considered as a problem, rather as an exciting challenge, except that our present-day knowledge of, especially deep-ocean, processes is rather shallow. Physical oceanographers have difficulty in putting numbers to the vertical flux of material (e.g., nutrients) into the photic zone; they cannot precisely predict the effects of varying climate on ocean circulation and sediment transport; they do not know whether hurricanes destroy living organisms and their ecology in the deep ocean.

Part of the problem is a lack of dedicated observations, to such extent that the phrase "We know more about the moon than the (deep) ocean" (e.g., Thar, 2011; Kershner, 2015) is still very much accurate. The difficulty lies in money spent, in coping with the rather harsh conditions of ambient pressure that increases by 1 Bar (one "atmosphere") every $10 \mathrm{~m}$, of the salt content and, in shallow waters, of sedimentological erosion and biological growth deteriorating instrumentation, while, in deeper waters, lack of light does not allow a single sun's photon to reach below 1,000 m. Making ocean observations is not straightforward, also because of the vastness of the ocean requiring careful planning before going out to sea, of the working from unstable platforms like ships riding surface waves, while modern electronics do not endure a single drop of sea water on their circuit boards. Satellite observations tell us a lot about the ocean's near-surface, but not much about the ocean interior. The ocean is far more transparent for acoustics than for light, but acoustics yield limited quantitative research on ocean dynamics as their reflectors vary in shape and density. Limited resources force the oceanographer to make observations in spatially one-dimensional 1D profiling, whether from a ship or using self-contained instrumentation moored between a buoy and an anchor at the bottom. 
It is thus not surprising that a substantial amount of research on geophysical flows, in particular on ocean dynamics driving the large- and small-scale circulation, has been performed in theory mainly, so far. In doing so, the limitations start with the presently still un-solvability (analytically) of the primary Navier-Stokes "NS" equations, which themselves form a rather comprehensive set. Although a good number of reasonable approximations are made for particular flows, the inherent nonlinearity of these equations is one of the outstanding problems in physics. It leads to perhaps the biggest challenge in classical physics, according to Einstein (and Feynman, https:// en.wikipedia.org/wiki/Turbulence) and very much valid to date, implying that no mathematical theory exists from first principles that describes nonlinear dynamics leading to turbulent flows that are omnipresent in geophysical flows. Without turbulence no marine life, no transport of matter, no ocean dynamics.

But, if a mathematical solution is in-existent one could nowadays resort to numerical simulations that, given the rapid development of modern electronics, may find a "reasonably accurate" approximate solution. Unfortunately, like clouds forming a big problem in (atmospheric) climate modeling and science (Beniston, 2013), we are still far from properly incorporating small-scale processes in the large-scale ocean circulation and even in regional models. In spite of tremendous improvements in computing skills over the last decades, we do not have a clue whether one wag of an anchovy's tail will alter the Kurushio Current or generate an underwater storm.

Apart from being able to understand certain individual processes, we have difficulty understanding more complex coupled processes. For example, the ocean may become warmer, but that does not necessarily directly imply that the stable density stratification increases. Stratification supports destabilizing shear to the point of marginal stability. This shear is largely imposed by internal waves supported by the same stratification, and which thus may help destroy their own habitat. As a result, we cannot yet predict the effects of ocean warming (or cooling) on vertical diapycnal exchange of suspended and resolved materials. Long-lived prejudice on particular ocean mechanisms hamper studies on potential coupling mechanisms, as it is quite likely that mechanisms on the large ocean basin scales (circulation), on meso-scales (eddies) and small-scales (turbulence) are all more or less connected.

\section{FUTURE CHALLENGES}

The ocean's governing physical processes of transport and redistribution of suspended and resolved materials are essentially three-dimensional at scales varying from $\mathrm{mm}$ to 1000 's of $\mathrm{km}$ spatially and from $0.01 \mathrm{~s}$ to years in time. Technically we still have a long way to go from present-day mainly $1 \mathrm{D}$ observations, even though satellites provide $2 \mathrm{D}$ imaging from the ocean surface. While the ocean's aspect ratio is approximately 1:1000, given the $3,700 \mathrm{~m}$ average depth and typical width of basins, mesoscale processes have 1:100 to 1:10 ratios and small-scale processes have scale ratios $>1: 10$ and into $1: 1$, the essential ratio for fully developed turbulence. To understand relevant ocean processes, we require information on the precise coupling between all scales. We need this information from observations that go beyond the $1 \mathrm{D}$, at least into $2 \mathrm{D}$, preferably full $3 \mathrm{D}$, and over long time scales while resolving the smallest time-scales.

One development is the installation of cabled networks at the ocean floor, with instruments that are powered from shore where they also send their data to that can be monitored in quasireal-time. This could be ideal for potential public participation research to cope with the expected large data sets, provided it is well guided. While the two principal means of investigating flows, at a fixed position in space "Eulerian," after the Swiss mathematician L. Euler, or following a particle and keeping track of its position "Lagrangean," after the French mathematician J. L. Lagrange, are defined since the Eighteenth century, practical sampling of physical oceanographic processes provides a more or less blurred image on the dynamics.

Moorings should not move to be Eulerian, which is physically impossible because the main drag and buoyancy forces do not balance as they are near-perpendicular to each other. During careful planning including minimizing drag by using thin cables and maximizing buoyancy one could arrive at weakly moving moorings by the large-scale current only, so that the Eulerian concept is well enough approximated.

Shipborne profiling, by the lowering of an instrument package at typical speeds of $0.5-1 \mathrm{~m} \mathrm{~s}^{-1}$ and mapping into quasi$2 \mathrm{D}$ by grouping several CTD-stations together in an ensemble requires a rather severe transformation or assumption of quasisynopticity to study basically steady-state or limited dynamical processes. Similarly, data sampled via autonomous vehicles cruising the oceans at typical speeds of about $0.25 \mathrm{~m} \mathrm{~s}^{-1}$, which are in the same range as general ocean flow speeds, can provide a cross-sectional overview of ocean properties but are difficult to transform to either Eulerian of Lagrangean frames of reference. Given their complex technical support needed it is not foreseen that such vehicle sampling will invalidate shipborne research in the near-future, also because ships remain needed to deploy other oceanographic equipment like moored instrumentation.

Although some success has been obtained using satellitetracked surface drifters to examine flows in a Lagrangean frame, a major challenge is the set-up of an ocean-interior Lagrangean experiment in which (a large set of) particles is followed, e.g. via a long baseline acoustic tracking system. While this is presently limited to small spatial scales only and perhaps in the future extendable to meso-scales, large ocean-basin scales are not expected to be resolved thus. Presently, the ocean-basin scales are resolved by an impressive network of some 3,800 Argo drifters (Argo, 2018), but these drifters are only tracked (via satellite) when they transmit their data after re-surfacing, every few days. Such observations are important for monitoring the state of the ocean, but are difficult to be used to understand the dynamical influence of the small- and meso-scales on the large-scale, in a proper Lagrangean framework.

While the all-scales research may prove a too large challenge observationally, the next step down (or up) is to study processes to such extent that they may be properly parameterized in ocean modeling. Such parametrizations can only be based on a thorough knowledge of the underlying physical processes. 
The advancement of computing power is so rapid that, still on relatively small scales, robust DNS modeling can progressively handle larger scales and more complex flows. (Direct Numerical Simulation numerically solves the full NS-equations at all scales albeit up to the computational limits, without a turbulence model). Nevertheless, the large Reynolds numbers typical for most ocean regions and implying the ubiquity of ocean turbulence are still (far?-) future challenge for most "realistic geometry" DNS. Work is also still in progress on the development of sub-grid scale DNS-models into less computationally costdemanding LES models that resolve only the large (eddy) scales. Perhaps a better set-up is to incorporate first LES into regional seas and large-scale ocean circulation models, as the effects of ocean eddies are not well understood on heat transport, for instance. Yet, simple numerical and analytical model analyses are needed to understand numerous existing small- and meso-scale problems.

While we are thus lacking a fundamental turbulence physics theory, several geophysical problems await a theoretical description. The precise transfer of energy from linear to nonlinear (internal) waves and their breaking is not solved. Boundary dynamics above sloping topography and wave breaking are different from frictional flow models above flat bottoms. The same for interaction between meso-scale eddies and their effect on ocean heat transport. While principal energy sources that drive the ocean are known, like the Sun, Earth rotation and tides, their precise interaction processes are key outstanding problems. The occurrence of extreme events has to be taken further than a statistical description. Such problems are

\section{REFERENCES}

Argo (2018). Available online at: http://www.argo.ucsd.edu/About_Argo.html

Beniston, M. (2013). Grand challenges in climate research. Front. Environ. Sci. 1:1. doi: 10.3389/fenvs.2013.

00001 Kershner, K. (2015). Do We Really Know More About Space Than the Deep? Available online at: https://science.howstuffworks.com/environmental/earth/ oceanography/deep-ocean-exploration.htm

Thar, J. (2011). Why Should We Know More About the Moon Than Our Oceans? The Vancouver Sun Available online at: http://blogs.vancouversun.com/2011/ 06/07/world-oceans-day-why-should-we-know-more-about-the-moonthan-our-oceans/ not only challenging for physical oceanography, but also for the impact on marine life and other disciplines.

\section{CONCLUSION}

The ocean seems vast and unknown, but although we do not fully understand its capability of feed-back systems to varying impacts there is a tendency toward general consensus that it is vulnerable. The ocean is not only vulnerable to pollution, in direct manners like plastic dumping and overfishing or in indirect manners like artificial heating, it is likely vulnerable to unknowns like ice sheet collapse and human extraction of (tidal) energy. Better knowledge of governing physical oceanographic processes is needed to argument against persistent prejudices. Some say the water motion contains "a lot of energy." However, there is relatively little energy in tides, by far not enough to cover human power-consumption, but it is crucial for various effects ranging from the maintenance of the ocean stratification and overturning circulation, via diapycnal turbulent mixing, to most important fishery nurseries in estuaries. Some say the Gulf Stream may change direction. However, the main drivers are not the ocean density variations but the Earth rotation and the wind, which will not be significantly saltered on short time scales (Wunsch, 2004).

\section{AUTHOR CONTRIBUTIONS}

The author confirms being the sole contributor of this work and has approved it for publication.

Wunsch, C. (2004). Gulf Stream safe if wind blows and Earth turns. Nature 428:601. doi: 10.1038/428601c

Conflict of Interest Statement: The author declares that the research was conducted in the absence of any commercial or financial relationships that could be construed as a potential conflict of interest.

Copyright (C) 2018 van Haren. This is an open-access article distributed under the terms of the Creative Commons Attribution License (CC BY). The use, distribution or reproduction in other forums is permitted, provided the original author(s) and the copyright owner(s) are credited and that the original publication in this journal is cited, in accordance with accepted academic practice. No use, distribution or reproduction is permitted which does not comply with these terms. 